\title{
Optical investigations of Berthelot-type properties in quaternary AlInGaN multiple quantum well heterosystems
}

\author{
Tzer-En Nee ${ }^{\mathrm{a}, *}$, Jen-Cheng Wang ${ }^{\mathrm{a}}$, Cheng-Wei Hung ${ }^{\mathrm{a}}$, Chih-Chun Ke ${ }^{\mathrm{a}}$, Hui-Tang Shen ${ }^{\mathrm{a}}$, Ya-Fen Wu ${ }^{\mathrm{b}}$, \\ Chang-Cheng Chuo ${ }^{c}$, Zheng-Hong Lee ${ }^{c}$, Ping-Lin Fan ${ }^{d}$, Joe-Air Jiang ${ }^{e}$

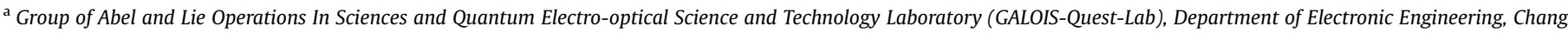 \\ Gung University, Kwei-Shan, Tao-Yuan 333, Taiwan, Republic of China \\ ${ }^{\mathrm{b}}$ Department of Electronic Engineering, Ming Chi University of Technology, Taishan, Taipei, Taiwan, Republic of China \\ ${ }^{c}$ Electronics and Optoelectronics Research Laboratories, Industrial Technology Research Institute, Hsin-Chu 300, Taiwan, Republic of China \\ d Department of Digital Technology Design and Graduate School of Toy and Game Design, National Taipei University of Education, Taipei 106, Taiwan, Republic of China \\ e Department of Bio-Industrial Mechatronics Engineering, National Taiwan University, Taipei 106, Taiwan, Republic of China
}

\section{A R T I C L E I N F O}

\section{Article history:}

Received 10 May 2007

Received in revised form

7 April 2009

Accepted 22 April 2009

Communicated by G.B. Stringfellow

Available online 5 May 2009

PACS:

85.60.Jb

78.67.De

78.55. $\mathrm{Cr}$

Keywords:

A3. Multiple quantum well

B2. Semiconductor quaternary alloys

B3. Light-emitting diodes.

\begin{abstract}
A B S T R A C T
The anomalous Berthelot-type optical properties of quaternary AlInGaN heterostructure with different quantum well pairs have been investigated systematically in this study. A microscopic model based on the luminescence observations of a comparison between an Arrhenius-type radiative process and a Berthelot-type nonradiative process has been proposed to elucidate the temperature dependence of the optical and transport behavior in nanocrystalline and amorphous semiconductors. The photoluminescence of the AlInGaN heterostructures is also found to exhibit unique luminescence features such as Sshaped emission peak energy and W-shaped full-width at half-maximum (FWHM) curves over a broad range of temperature. We investigated in detail the aspects of luminescence that would provide significant information regarding the degree of disorder in AlInGaN-based semiconductor heterosystems, leading to the appearance of the Berthelot-type behavior. The increase of quantum-well pairs apparently will cause the incorporation of indium atoms in the AlInGaN nanostructures, resulting in augmentation of the degree of crystalline randomization. In other words, the higher degree of disorder in AlInGaN heterostructures, the longer the static microbarrier width and the more obvious carrier localization effects may be observed.
\end{abstract}

Crown Copyright (c) 2009 Published by Elsevier B.V. All rights reserved.

\section{Introduction}

Quaternary AlInGaN semiconductors have recently attracted much attention, especially for their potential applications in deepultraviolet light-emitting diodes (LEDs) and ultraviolet-blue laser diodes (LDs) [1,2] for long-lifetime lighting, medical devices, and high-density optical storage, as well as for illumination [3]. Despite the striking improvements that quaternary AlInGaNbased optoelectronic devices have achieved, the influence of the solid-phase indium miscibility gap on the optical properties remains a debatable issue [4]. Also, due to the alloy disorder and interface irregularities, this gap has been associated with disordering by the composition fluctuations of indium content.

A microscopic model based on the luminescence observations of competition between an Arrhenius-type radiative process and Berthelot-type nonradiative process has been proposed to elucidate the temperature dependence of the optical and transport

\footnotetext{
*Corresponding author. Tel.: +88632118800; fax: +88632118507
}

E-mail address: neete@mail.cgu.edu.tw (T.-E. Nee). behavior in nanocrystalline and amorphous semiconductors [5]. Due to alloy spinodal decompositions, the photoluminescence (PL) and electroluminescence (EL) spectra of the quaternary AlInGaN, ternary AlGaN/GaN, and ternary InGaN/GaN heterostructures have been found to exhibit unique luminescence features such as S-shaped emission peak energy (redshift-blueshift-redshift) and W-shaped full-width at half-maximum (FWHM) curves over a broad range of temperature, leading to the appearance of Berthelot-type behavior [5].

The luminescence spectra from the GaN-based multiple quantum well (MQW) active regions may be assigned to a recombination of excitons, localized at potential minima, which originates from In-rich regions acting as quantum dots [6]. However, little work has been done to elaborate the presence of In-rich regions in InGaN layer acts as localization centers that trap the carriers into the quantum-well vicinities $[7,8]$. The temperature-induced S-shaped PL shift is strongly affected by the competition of carrier recombination mechanisms with increasing temperature. In the low-temperature region, the redshift of the peak energy is attributed to carriers having more opportunity to relax into the lower-energy tail states caused by 
the inhomogeneous potential fluctuations. The reason is that the carrier lifetime tends to increase as the temperature rises [9]. Furthermore, the temperature-induced blueshift of the emission peak can be demonstrated by the band-tail model with a Gaussian-like distribution of the density of states and the screening effect of the piezoelectric field $[10,11]$.

Recently, Berthelot-type mechanisms have been proposed to characterize the anomalies of the temperature-induced spectral blueshift in nanostructure materials of elementary and compound semiconductors [12]. In this study, we investigate the nature of the spectral behavior of the luminescence emission peak and emphasize the observation of the temperature-induced shift, wherein significant information on the degree of disorder in AlInGaN quaternary systems may be found. We characterized the spectral properties of the multiple quantum well $(\mathrm{p}-\mathrm{i}-\mathrm{n})$ heterostructure, composed of AlInGaN heterobarriers with different quantum-well pairs for a temperature range from 20 to $300 \mathrm{~K}$. Therefore, we observed that augmenting the degree of disorder in heterosystems leads to both the extension of static microbarrier width, and the enhancement of carrier localization effects. The purpose of this investigation is not only to refer the quaternary AlInGaN to disorder material system, but also to propose that Berthelot-type model may be used to examine the abnormal luminescence spectra.

\section{Experiments}

The samples investigated in this study were grown by metalorganic vapor-phase epitaxy (MOVPE) on c-plane sapphire substrates with a 25-nm-thick, low-temperature GaN nucleation layer. The sample structure consisted of a 3- $\mu$ m-thick n-type GaNdoped layer, a 3- $\mu$ m-thick n-type AlGaN layer, a three-, five-, and ten-period undoped quaternary AlInGaN MQWs active layer, followed by a $0.15-\mu$ m-thick p-AlGaN contact layer. Silane $\left(\mathrm{SiH}_{4}\right)$ and bis(cyclopentadienyl)magnesium $\left(\mathrm{Cp}_{2} \mathrm{Mg}\right)$ were used as either the n-type and p-type precursors or dopants, respectively. The doping concentration was $5 \times 10^{18} \mathrm{~cm}^{-3}$ for the former and $1 \times 10^{19} \mathrm{~cm}^{-3}$ for the latter. Each AlInGaN MQW pair consists of a 2-nm-thick well layer and a 10-nm-thick barrier layer. For temperature-dependent photoluminescence measurements, samples were mounted in a closed-cycle He cryostat and excited by a continuous-wave He-Cd laser, while the temperature gradually increased from 20 to $300 \mathrm{~K}$. The average excitation intensity was $20 \mathrm{~mW}$. The luminescence signal was dispersed through a $0.5 \mathrm{~m}$ monochromator and later detected by a Si photodiode, during which a standard lock-in amplification technique was employed.

\section{Results and discussion}

Fig. 1 shows the evolution of PL emission peak energy for the AlInGaN heterostructures with three, five, and ten pairs of wells over a temperature range from 20 to $300 \mathrm{~K}$. As can be seen, the emission peak energy positions of the AlInGaN semiconductor exhibit S-shaped curves (redshift-blueshift-redshift) when the temperature increases. To assess the mole fraction of elements, we analyzed the content of indium from X-ray diffraction (XRD) and the Varshni formula including emendatory term based on the band-tail model. The approximate compositions for the samples with different pairs are listed in Table 1, which shows a clear indication that In composition increases with the pairs of quantum wells; namely, the AlInGaN sample with a higher quantum-well pair has the nanocrystallites with higher In composition. Due to the carrier lifetime being prolonged by increasing temperature, we discovered that the PL peak energy

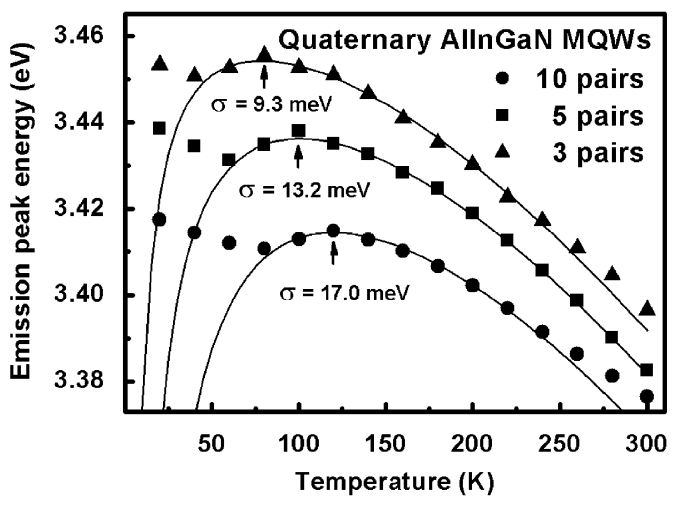

Fig. 1. The photoluminescence emission peaks are function of temperature for the quaternary AlInGaN MQW structures with three, five, and ten pairs of quantum wells. The solid curves under the symbols are theoretical fitted results derived from the Varshni equation and band-tail model. The labeled arrows indicate the turning-point temperatures for emission transition of the three samples.

Table 1

The compositions of $\mathrm{Al}_{x} \mathrm{In}_{y} \mathrm{Ga}_{1-x-y} \mathrm{~N}$ heterostructures were extracted from X-ray diffraction measurement and Varshni formula that includes band-tail model analyses.

\begin{tabular}{cccccc}
\hline MQW pairs & In (\%) & $\mathrm{Al}(\%)$ & $\mathrm{Ga}(\%)$ & $\sigma(\mathrm{meV})$ & $E_{a}(\mathrm{meV})$ \\
\hline 3 & 3.67 & 6.13 & 90.20 & 9.30 & 13.5 \\
5 & 3.90 & 6.15 & 89.95 & 13.2 & 14.9 \\
10 & 4.22 & 6.15 & 89.63 & 17.0 & 21.1 \\
\hline
\end{tabular}

The $\sigma$ indicates the degree of localization effect extracted from the emission peak energy as a function of temperature for the quaternary AlInGaN MQW structures by the Varshni formula and band-tail model. The activation energies were extracted from Arrhenius plot of temperature-dependent photoluminescence intensity for the quaternary AlInGaN MQW structures.

values decrease when the temperature increases from 20 to $40 \mathrm{~K}$ for the three-pair sample, from 20 to $60 \mathrm{~K}$ for the five-pair sample, and from 20 to $80 \mathrm{~K}$ for the 10 -pair sample. By further increasing the temperature, the spectral blueshift occurred at temperatures lower than the turning-point temperature (designated as $T_{t}$ ), which is dominated by the combined effects of a strain-induced quantum-confined Stark shift and band filling [13,14], leading to the appearance of Berthelot-type behavior. Furthermore, as the arrows show in Fig. 1, the observed $T_{t}$ for the quaternary AlInGaN MQW structures with three, five, and ten pairs of wells are around 80,100 , and $120 \mathrm{~K}$, respectively. We found that the turning-point temperature increases and the temperature-induced blueshift change of emission peak energy decreases with rising quantumwell pairs. With temperature increased up to $300 \mathrm{~K}$, the redshifttemperature evolutions of the emission peak energy values for all samples are notable and consistent with temperature-induced bandgap shrinkage of the quaternary AlInGaN MQW structure configurations.

To estimate the changes of peak energy in Fig. 1, based on the Varshni formula and band-tail model in Eq. (1), the temperaturedependent emission energy can be calculated by the following expression [15]:

$E_{g}(T)=E_{g}(0)-\frac{\alpha T^{2}}{T+\beta}-\frac{\sigma^{2}}{k_{B} T}$,

where $T$ is temperature in Kelvin, $E_{g}(0)$ describes the energy gap at zero temperature, and $\alpha$ and $\beta$ are known as Varshni's fitting parameters. The third term on the right in the equation originates from the localization effect, in which $\sigma$ indicates the degree of localization effect, i.e., a large value of $\sigma$ means a strong 
localization effect; $k_{B}$ is Boltzmann's constant. The parameters obtained from the approximate fit are $\alpha=0.97 \mathrm{meV} / \mathrm{K}$ and $\beta=810 \mathrm{~K}$ for the samples. By using Eq. (1), we can extract the value of $\sigma$ and realize the degree of quantum exciton localization effect. Through the theoretical fits, we obtain values of $\sigma$ for the quaternary AlInGaN MQW structures with three, five, and ten pairs of wells as roughly $9.3,13.2$, and $17.0 \mathrm{meV}$, respectively. Little work has been done to elaborate the presence of In-rich regions in InGaN layer acts as localization centers that trap the carriers into the quantum-well vicinities $[7,8]$. The fact of strong emission from InAlGaN in comparison with that of AlGaN is considered to be due to the fact that the excited carriers move into the In-rich region and recombine radiatively rather than be trapped in the nonradiative centers generated by defects [16]. However, the variation in potential fluctuation and indium segregation may be caused by the variation in dislocation density and interface roughness with an increasing number of wells [11]. From the element composition list in Table 1, we observed that the spatial indium fluctuations enriched and the exciton localization effect gradually enhanced with increasing pairs of quantum wells, resulting from indium segregation regions acting as quantumdot-like structures. Therefore, we consider that the emission fluctuation might be due to carrier localization in the Insegregation area. Furthermore, the PL peak shifts are due mainly to potential fluctuation caused by In composition fluctuation and interface roughness rather than the piezoelectric filed.

For an in-depth understanding of how photo-excitation and compositional inhomogeneity influence emission peak position, we examined the characteristics of the unique optical behavior by evaluating the evolution of $T_{t}$. Employing the analytical formulations developed by John and Singh [17], the bandgap $\left(E_{g}\right)$ is assumed to decrease linearly as temperature rises. We know that the AlInGaN bandgaps will shrink with temperature increase; therefore, the temperature dependence of the anomalous optical properties can be expressed as [17]

$\frac{E_{p h}-E_{g}}{T}=-C_{1}-\frac{C_{2}}{T^{3}}$,

where $E_{p h}$ is the photon energy, $E_{g}$ the energy bandgap at $0 \mathrm{~K}, C_{1}$ the thermal coefficient of the bandgap with respect to temperature, and $C_{2}$ a structural parameter associated with the degree of disorder. The crossover temperature $\left(T_{t}\right)$ can be extracted numerically by using the expression $T_{t}=\left(2 C_{2} / C_{1}\right)^{1 / 3}$ [17]. We expect, however, that the value of $C_{1}$ will be constant, regardless of the heterostructure of the device. With the intention of exploring the dominance of the photo-excitation in the value of $T_{t}$, the temperature derivative of Eq. (2) can be derived

$\frac{d}{d T} \frac{\left(E_{p h}-E_{g}\right)}{T}=\frac{3 C_{2}}{T^{4}}$

also, the $C_{2}$ value is related to the tunneling statistic microbarrier width and the carrier vibrating frequency such as Eq. (4)

$C_{2}=\frac{\hbar^{2}}{2 m}\left(\frac{S M \Omega^{2}}{2 k}\right)^{2}$,

where $S$ is the tunneling statistic microbarrier width, $M$ the inertia of the vibrating system, and $\Omega$ represents the hopping frequency.

From the slopes of the plots of $d\left(E_{p h}-E_{g}\right) T^{-1} / d T$ vs. $3 T^{-4}$ (Fig. 2), we can obtain the respective values of $C_{2}$ for the three various pairs of the sample over a broad temperature range. Under the same photo-excitation density, the ten pairs of MQW samples with a larger value of $C_{2}$ exhibit a high value of $T_{t}$ as well over the temperature range. This is because the thermally excited carriers may have enough energy to hop in the quantum-dot-like region of the nanocrystalline. As the Berthelot-type model is derived, the

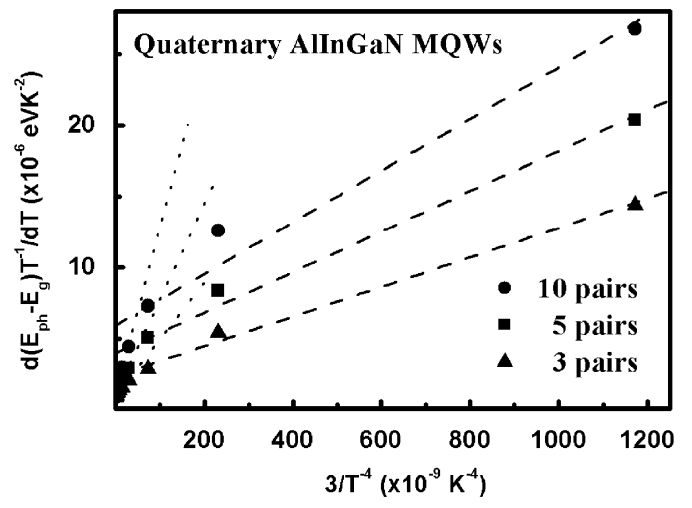

Fig. 2. Plots of $d\left(E_{p h}-E_{g}\right) T^{-1} / d T$ versus $T^{-4}$ for the positions of the temperaturedependent PL peak energies of the quaternary AlInGaN MQW structures with three, five, and ten pairs of quantum wells. The measured data are indicated using the symbols; the dash lines are the theoretical fitted results using the Berthelottype model.

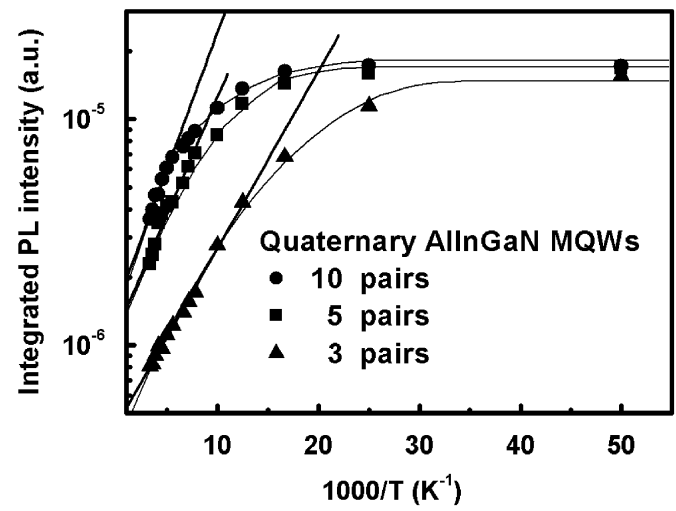

Fig. 3. The Arrhenius plot of the integrated PL intensity of emission states for the quaternary AlInGaN MQW structures with three, five, and ten pairs of quantum wells.

turning temperature is proportional to the square of the statistic microbarrier width and fourth power of the vibrating process frequency. Under low temperature, the value of $C_{2}$ is dominated by the broader microbarrier width because electron-hole pairs are almost frozen in the epitaxial layers without vibration. When the temperature is raised further, carriers with higher hopping frequency become the dominate factor influencing the value of $C_{2}$. Therefore, the magnitude of $C_{2}$ value could be larger under the high-temperature range. According to the disorder model hypothesis, the value of $T_{t}$ associated with the heteropotential configuration rises directly with respect to microbarrier width [5]. Nevertheless, the spatial localization and phase separation of Inrich clusters in quaternary AlInGaN quantum structures causes the broadening of the excitonic emission of the alloy structures. Because of the inhomogeneities in composition, potential energy fluctuations, and structure variation with and without alloy disorder, the result is a spatially random and energetically disordered system of carrier localization. Therefore, the increases in the MQW pairs enhanced the localization of the excitonic resonances, and decreased the probability of the carrier tunnel/hopping process.

Fig. 3 shows an Arrhenius plot of the integrated PL intensity of emission state for the quaternary AlInGaN MQW structures with three, five, and ten pairs of wells, over a broad temperature range. The quenching of the PL luminescence with temperature is attributed to the thermal emission of the photocarriers that 
escaped the local potential minima caused by potential fluctuations, such as alloy and interface fluctuations. Hence, the activation energy is correlated with the degree of the exciton confinement. The following expression is generally used to calculate the activation energy $\left(E_{A}\right)$ in thermally activated processes [18]:

$$
I(T)=\frac{I_{0}}{1+A \exp \left(-E_{A} / k_{B} T\right)}
$$

where $I(T)$ is the temperature-dependent integrated PL intensity, $I_{0}$ the integrated PL intensity at $0 \mathrm{~K}, k_{B}$ Boltzmann's constant, and $A$ rate constant. The $E_{A}$ values for the three, five, and ten pairs of quaternary AlInGaN MQW structures extracted from the lowexcitation Arrhenius plots in Fig. 3 are 13.5, 14.9, and $24.1 \mathrm{meV}$, respectively. The luminescence spectra from the GaN-based multiple quantum well active regions may be assigned to a recombination of excitons, localized at potential minima, which originates from In-rich regions acting as quantum dots [6]. The activation energies are often referred to as the minimal energies required for most carriers to escape from the localized states. Higher activation energy enables the energetic carriers be circumscribed in the active region. However, the exciton wavefunction can be successfully tailored by the nanostructure, which facilitates the localization of the injected carriers, as well as promotes radiative recombination in the active region and inhibits the rapid degradation of luminescence. Consequently, the experimental findings imply that quaternary AlInGaN MQW nanostructures with lower In-content nanocrystallites and narrower static microbarrier width may provide alternative paths for radiationless transitions.

Fig. 4 shows the temperature-dependent linewidths of the emission states for the three samples, which are obtained by extracting these luminescence spectra with a Gaussian fit at the same photo-excitation levels. The full-width at height-maximum

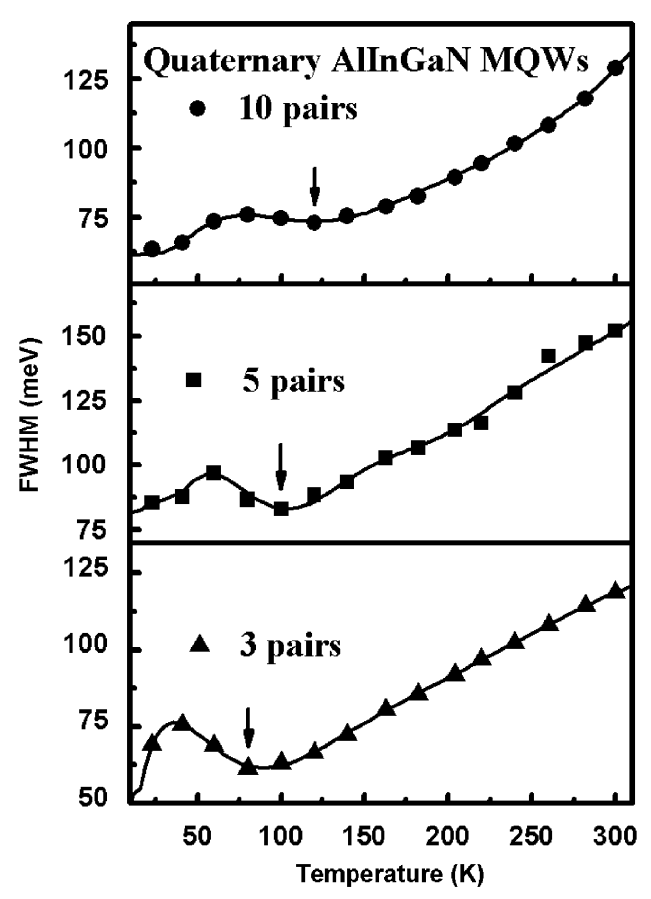

Fig. 4. Linewidths of emission peak versus temperatures for the quaternary AlInGaN MQW structures with three, five, and ten pairs of quantum wells. The labeled arrows indicate the turning-point temperatures for emission transition of the three samples.
(FWHM) is generally used to verify variation of the emission exciton from different energy states, i.e., the more emission exciton from different energy states, the higher the degree of disorder. From the PL spectra of the samples, we observed that the linewidth and wavelength of the emission state for these samples decrease when the temperatures are lower than $T_{t}$, while those increase when temperature goes beyond $T_{t}$, leading to the appearance of Berthelot-type behavior. As the temperature rises, the linewidth of the quaternary AlInGaN-related emission exhibits a W-shaped curve due to the crossover from nonthermalized to thermalized distribution of localized excitons through the carrier transport and hopping process [19]. Therefore, the exciton hopping strongly between isolated In-rich clusters with a much smoother potential energy profile and more interface roughness due to the fluctuations of indium composition. Furthermore, it also led the carriers to transport randomly form the spaced localization sites over the well vicinities.

For indium segregation caused randomize distribution in quaternary AlInGaN heterostructures, the temperature-induced carrier-transfer mechanism is contingent on the static microbarrier and carrier dynamic frequency. We observed that augmenting the degree of disorder in heterosystems leads to both the extension of static microbarrier width, and the enhancement of carrier localization effects. In other words, the higher degree of disorder in AlInGaN heterostructures, the longer the static microbarrier width and the more obvious carrier localization effects may be observed.

On the other hand, the higher In-content quaternary AlInGaN sample is expected to assist the carriers drifting coherently across the crystallite microbarriers, while also causing strong excitonrecombination accumulation at local potential minima. The origin of strong emission from AlInGaN is attributed to the fact that the excited carriers move into the In-rich region and recombine radiatively rather than be trapped in the nonradiative centers generated by defects. Both effects derive from a microcrystalline randomization, leading to the corresponding high $T_{\mathrm{t}}$ in the Berthelot-type process. The purpose of this investigation is not only to refer the quaternary AlInGaN to disorder material system, but also to propose that Berthelot-type model may be used to examine the abnormal luminescence spectra.

\section{Conclusion}

In this study, Berthelot-type optical properties of AlInGaN structures with different pairs of MQWs have been investigated systematically. We found that the higher the turning-point temperature, the higher the degree of crystalline randomization on quaternary AlInGaN heterosystems, due to the increase in the incorporation of indium atoms in nanostructures. The abnormal emission peak energy evolution is found to be temperature dependent, and the characteristic turning temperatures derived from Berthelot-type are between 80 and $120 \mathrm{~K}$. The linewidth of the quaternary AlInGaN-related emission exhibits a W-shaped curve due to the crossover from nonthermalized to thermalized distribution of localized excitons through the carrier transport process, which results in carriers transport randomly form the spaced localization sites over the well vicinities. These phenomena are attributed to the variations of effective indium composition increases with the pairs of quantum wells; namely, the AlInGaN sample with a higher quantum-well pair has the nanocrystallites with higher In composition. Therefore, we observed that augmenting the degree of disorder in heterosystems leads not only to the extension of static microbarrier width, but also to the enhancement of carrier localization effects. 


\section{Acknowledgment}

This work is supported by the National Science Council of the Republic of China under Contract no. NSC 97-2112-M-182-002MY3.

\section{References}

[1] M. Shatalov, J. Zhang, A.S. Chitnis, G. Simin, M.A. Khan, IEEE J. Quantum Electron. 8 (2002) 2.

[2] S. Nagahama, T. Yanamoto, M. Sano, T. Mukai, Jpn. J. Appl. Phys. 40 (2001) L788.

[3] H. Hirayama, J. Appl. Phys. 97 (2005) 091101.

[4] R. Singh, D. Doppalapudi, T.D. Moustakas, L.T. Romano, Appl. Phys. Lett. 70 (1997) 1089.

[5] T.E. Nee, H.T. Shen, J.C. Wang, R.M. Lin, J. Cryst. Growth 287 (2006) 468.

[6] H. Hirayama, A. Kinoshita, T. Yamabi, Y. Enomoto, A. Hirata, T. Araki, Y. Nanishi, Y. Aoyagi, Appl. Phys. Lett. 80 (2002) 207.

[7] M.C. Cheung, G. Namkoong, F. Chen, M. Furis, H.E. Pudavar, A.N. Cartwright, W.A. Doolittle, Phys. Status Solidi (c) 2 (2005) 2779.
[8] C.A. Tran, R.F. Karlicek Jr., M. Schurman, A. Osinsky, V. Merai, Y. Li, I Eliashevich, M.G. Brown, J. Nering, I. Ferguson, R. Stall, J. Cryst. Growth 195 (1998) 397.

[9] Y. Cho, G.H. Gainer, A.J. Fischer, J.J. Song, S. Keller, U.K. Mishra, S.P. DenBaars, Appl. Phys. Lett. 73 (1998) 1370.

[10] P.G. Eliseev, J. Appl. Phys. 93 (2003) 5404

[11] H.-K. Yuh, E. Yoon, S.K. Shee, J.B. Lam, C.K. Choi, G.H. Gainer, G.H. Park, S.J. Hwang, J.J. Song, J. Appl. Phys. 91 (2002) 3483.

[12] K. Kazlauskas, G. Tamulaitis, A. Zukauskas, M.A. Khan, J.W. Yang, J. Zhang, E. Kuokstis, G. Simin, M.S. Shur, R. Gaska, Appl. Phys. Lett. 82 (2003) 4501.

[13] S.F. Chichibu, A.C. Abare, M.S. Minsky, S. Keller, S.B. Fleischer, J.E. Bowers, E. Hu, U.K. Mishra, L.A. Coldren, S.P. DenBaars, T. Sota, Appl. Phys. Lett. 73 (1998) 2006.

[14] M. Ryu, C.Q. Chen, E. Kuokstis, J.W. Yang, G. Simin, M.A. Khan, Appl. Phys. Lett. 80 (2002) 3730.

[15] T. Wang, P.J. Parbrook, M.A. Whitehead, Phys. Status Solidi (c) 0 (2003) 2019

[16] H. Hirayama, Y. Enomoto, A. Kinoshita, A. Hirata, Y. Aoyagi, Appl. Phys. Lett. 80 (2002) 1589.

[17] G.C. John, V.A. Singh, Phys. Rev. B 54 (1996) 4416.

[18] J. Li, K.B. Nam, K.H. Kim, J.Y. Lin, H.X. Jiang, Appl. Phys. Lett. 78 (2001) 61.

[19] K. Kazlauskas, G. Tamulaitis, A. Zukauskas, M.A. Khan, J.W. Yang, J. Zhang, G. Simin, M.S. Shur, R. Gaska, Phys. Status Solidi (c) 0 (2002) 512. 